\title{
Demonstrating Learning of Register Automata
}

\author{
Maik Merten ${ }^{1}$, Falk Howar ${ }^{1}$, Bernhard Steffen ${ }^{1}$, Sofia Cassel$^{2}$, \\ and Bengt Jonsson ${ }^{2}$ \\ 1 Technical University Dortmund, Chair for Programming Systems, Dortmund, \\ D-44227, Germany \\ \{maik.merten, falk.howar, steffen\}@cs.tu-dortmund.de \\ 2 Dept. of Information Technology, Uppsala University, Sweden \\ \{sofia.cassel, bengt.jonsson\}@it.uu.se
}

\begin{abstract}
We will demonstrate the impact of the integration of our most recently developed learning technology for inferring Register $\mathrm{Au}-$ tomata into the LearnLib, our framework for active automata learning. This will not only illustrate the unique power of Register Automata, which allows one to faithfully model data independent systems, but also the ease of enhancing the LearnLib with new functionality.
\end{abstract}

\section{Introduction}

Active automata learning (aka regular extrapolation) has been proposed to semiautomatically infer formal behavioral models of underspecified systems. The resulting formal behavioral models can be used, e.g., for documentation or regression testing and thus can be an enabler for continued system evolution. Automated mediation between networked systems by automatically synthesizing connectors from behavioral models is a current research interest. This approach is currently in development in the CONNECT project [6].

To cater the various use-cases of automata-learning, LearnLib has been created to offer a versatile library of learning algorithms and related tools. Result of an extensive reengineering effort, the Next Generation LearnLib [8] implements a flexible component-based approach that supports quick iteration and refinement of learning setups (in the following the Next Generation LearnLib will simply be referred to as "LearnLib").

The reengineered LearnLib has seen continued evolution, for which a complete account will not be provided in this paper. We will rather focus on two main innovations:

- The modeling paradigm in LearnLib Studio underwent significant changes. For example, in the old modeling paradigm a dedicated setup phase would precede the actual learning process. This has been replaced by on-the-fly configuration during the learning phase itself, with only minimal static configuration needed beforehand.

\footnotetext{
* This work is supported by the European FP 7 project CONNECT (IST 231167). 
- LearnLib has been outfitted with support for the Register Automata automaton model [4, which is a simple extension of finite automata with data from infinite domains. It can model data-independent systems [7, i.e., systems that do not compute or manipulate data but manage their adequate distribution, such as protocols and mediators, in an intuitive way.

The demonstration will not only cover these two points in isolation, but will also highlight the ease of integration of new functionality into the overall learning framework.

In the remainder of the paper, we will recall the basics of active automata learning in Section 2, followed by an introduction to Register Automata in Section 3. A learning solution for Register Automata will be presented in Section 4 . demonstrating the innovations outlined above. In Section 5 a conclusion is provided, with references showcasing the broad application scope of the presented tool environment.

\section{Active Automata Learning}

Angluin's seminal algorithm $L^{*}$ [2] defines two query types to gather information about the System Under Learning (SUL):

- Membership Queries (MQs) are traces of symbols from a predefined alphabet of inputs of the SUL. The learning algorithm will construct such input traces, execute these on the SUL and capture system output. From the gathered information a hypothesis model is generated.

- Equivalence Queries (EQs) compare the produced hypotheses with the target system. If the model is not accurate, a counterexample will be provided revealing a difference between the current hypothesis and the SUL. Evaluating counterexamples the learning algorithm will produce refined hypothesis models using additional MQs. Once no counterexample can be produced the learning procedure has produced an accurate model and can be stopped.

With those two query types, $L^{*}$ is guaranteed to produce a minimal and correct model. In current practice, however, EQs can only be implemented approximately for a large class of systems, e.g., with additional invocations of the target system.

The original $L^{*}$ algorithm has originally been presented for DFAs, but has since been adapted to Mealy Machines, which are a better fit for learning actual reactive systems as they can encode system output in a natural way. A major and recent increase in expressiveness is achieved with Register Automata [5], which are described in the following section.

\section{Register Automata}

Register Automata are an extension of finite automata with data from infinite domains and are, e.g., well-suited for describing communication protocols. Register Automata are defined as follows: 


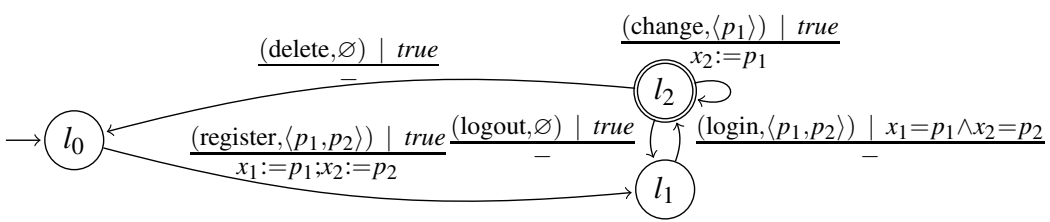

Fig. 1. Partial RA model for a fragment of XMPP

Definition 1. Let a symbolic input be a pair $(a, \bar{p})$, of a parameterized input $a$ of arity $k$ and a sequence of symbolic parameters $\bar{p}=\left\langle p_{1}, \ldots, p_{k}\right\rangle$ Let further $X=\left\langle x_{1}, \ldots, x_{m}\right\rangle$ be a finite set of registers. A guard is a conjunction of equalities and negated equalities, e.g., $p_{i} \neq x_{j}$, over formal parameters and registers. An assignment is a partial mapping $\rho: X \rightarrow X \cup P$ for a set $P$ of formal parameters.

Definition 2. A Register Automaton (RA) is a tuple $\mathcal{A}=\left(A, L, l_{0}, X, \Gamma, \lambda\right)$, where

- $A$ is a finite set of actions.

$-L$ is a finite set of locations.

$-l_{0} \in L$ is the initial location.

$-X$ is a finite set of registers.

$-\Gamma$ is a finite set of transitions, each of which is of form $\left\langle l,(a, \bar{p}), g, \rho, l^{\prime}\right\rangle$, where $l$ is the source location, $l^{\prime}$ is the target location, $(a, \bar{p})$ is a parameterized action, $g$ is a guard, and $\rho$ is an assignment.

$-\lambda: L \mapsto\{+,-\}$ maps each location to either + (accept) or - (reject).

Let us define the semantics of an $\mathrm{RA} \mathcal{A}=\left(A, L, l_{0}, X, \Gamma, \lambda\right)$. A $X$-valuation, denoted by $v$, is a (partial) mapping from $X$ to $D$. A state of $\mathcal{A}$ is a pair $\langle l, v\rangle$ where $l \in L$ and $v$ is a $X$-valuation. The initial state is $\left\langle l_{0}, v_{0}\right\rangle$, i.e., the pair of initial location and empty valuation.

A step of $\mathcal{A}$, denoted by $\langle l, v\rangle \stackrel{(a, \bar{d})}{\longrightarrow}\left\langle l^{\prime}, v^{\prime}\right\rangle$, transfers $\mathcal{A}$ from $\langle l, v\rangle$ to $\left\langle l^{\prime}, v^{\prime}\right\rangle$ on input $(a, \bar{d})$ if there is a transition $\left\langle l,(a, \bar{p}), g, \rho, l^{\prime}\right\rangle \in \Gamma$ such that $(1) g$ is modeled by $\bar{d}$ and $v$, i.e., if it becomes true when replacing all $p_{i}$ by $d_{i}$ and all $x_{i}$ by $v\left(x_{i}\right)$, and such that $(2) v^{\prime}$ is the updated $X$-valuation, where $v^{\prime}\left(x_{i}\right)=v\left(x_{j}\right)$ wherever $\rho\left(x_{i}\right)=x_{j}$, and $\mathrm{v}^{\prime}\left(x_{i}\right)=d_{j}$ wherever $\rho\left(x_{i}\right)=p_{j}$.

An example instance of a Register Automaton is provided in Figure 1, which models a subset of the XMPP instant messaging protocol focused on aspects of user authentication. In location $l_{0}$ no user account exists. With the parameterized action register a new account can be created, with the parameters $p_{1}$ and $p_{2}$ denoting a username and password. When executing the register action, the parameter values are copied into the registers $x_{1}$ and $x_{2}$ respectively. This action is unconditionally invocable in $l_{0}$, meaning that its guard is true. In contrast, the login in action of $l_{1}$ has a guard that specifies that the parameters $p_{1}$ and $p_{2}$ provided with the login action have to match the register contents of $x_{1}$ and $x_{2}$, i.e., the credentials provided during login have to match the ones stored in the registers. The other system actions represent logging out, changing the account password, and deleting the user account. 


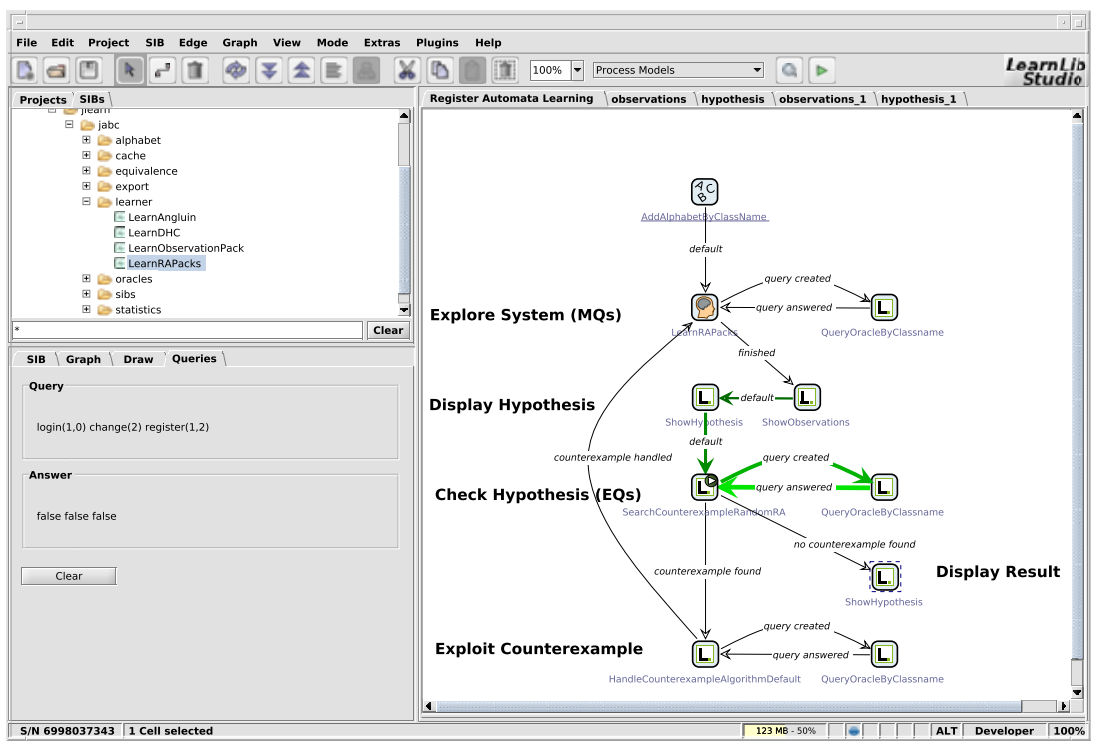

Fig. 2. A modeled learning setup created in LearnLib Studio. The model is currently executed, with bold edges denoting the path of execution. The current query and its answer are made visible in the panel on the lower left side.

\section{The Tool Demo}

We will present the LearnLib framework and highlight recently integrated innovations. LearnLib Studio allows the model-based composition and execution of learning setups, where LearnLib components are made available as reusable building blocks. In Figure 2 a learning setup tooled for learning register automata is shown, configured to learn the system shown in Figure 1. The result of executing this learning setup is presented in Figure 3.

The reworked modeling approach: In the learning setup presented in Figure 2 the distinct pattern specific to active automata learning can be witnessed: a learning algorithm is invoked, resulting in MQs an "oracle" has to answer. In this context, oracles are components that execute queries on a target system and gather the invocation results, meaning they produce an answer for a given query. Once the learning algorithm has formed a hypothesis it can be displayed and the data structure gathering the observations can be visualized, e.g., for debugging purposes. In the displayed setup, EQs are approximated by a random walk conformance test, which generates additional queries that are answered by the system oracle. If no counterexample can be found, the learning procedure will terminate, displaying the final result. Otherwise the counterexample will be consumed and analyzed, which can result in the production of additional queries. Once these have been answered and the counterexample is exploited so that a refined hypothesis can be produced, the learning process will restart the learning algorithm. 


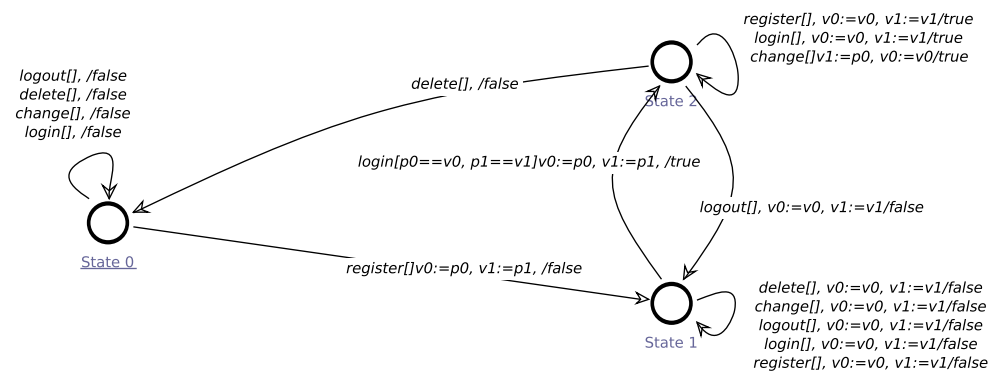

Fig. 3. The resulting model from executing the learning setup. Guards are in square brackets, the next register contents are explicitly denoted on every transition as is the acceptance status of the following state. Register values are denoted as $v_{i}$.

The general workflow is reusable in nature. The only application-specific parts that have to be provided are the definition of the alphabet and the system oracle, which has to interface the SUL. These application-specific parts can be loaded in a standardized way with parameterized building blocks.

Compared to the previous modeling style supported by LearnLib Studio, the new modeling style is much improved in terms of usability and flexibility: in the old modeling paradigm, a dedicated setup phase would create a fixed configuration, connecting, e.g., the learning algorithm to the SUL oracle. This setup would then be instantiated and started, with limited ways to influence the behavior of the setup afterwards. In the new modeling paradigm, only an alphabet has to be specified beforehand, after which the learning algorithm can directly begin operation. Learning queries are delegated on-the-fly according to the setup execution flow. This greatly increases flexibility, as, for instance, setups can decide at runtime what oracle instances are used to answer queries.

Integration of Register Automata learning: Thanks to the flexible component-based approach of LearnLib, components for learning this new model type could be integrated into the general framework without changes to the LearnLib architecture.

In the learning setup presented above, the only components that are specific for the RA machine model are the building blocks encapsulating the learning algorithm and the equivalence approximation. The overall infrastructure provided by the LearnLib is reused to a high degree when using RAs despite adapting a much richer automata model. The overall flavor of how learning setups can be created is completely unchanged compared to how setups are specified for other machine models, abstracting from the details of the underlying data structures and algorithms and thus shielding the user from additional complexity.

Due to being integrated into the component framework, learning setups for Register Automata can use all facilities LearnLib offers for debugging, visualization and statistics. Thus our extension provides a powerful and unique framework for learning data independent systems [7. 


\section{Conclusion}

The LearnLib framework and accompanying tools provide a rich environment for experimentation with the new Register Automata formalism. Embedded into a flexible component model, much functionality is shared between learning setups for different machine models, which enables a high degree of reuse. Users already versed in the operation of the LearnLib Studio will not have to learn a new style of modeling when adapting Register Automata, while users new to LearnLib Studio are only exposed to a limited set of generic concepts that are easy to understand.

The LearnLib is mature and used by several independent research groups. It has, e.g., been used to infer the behavior of a electronic passports [1, in security research [3, and it is a central enabler within the CONNECT framework.

LearnLib is available for download at http://www.learnlib.de and free for all academic purposes.

\section{References}

1. Aarts, F., Schmaltz, J., Vaandrager, F.: Inference and Abstraction of the Biometric Passport. In: Margaria, T., Steffen, B. (eds.) ISoLA 2010, Part I. LNCS, vol. 6415, pp. 673-686. Springer, Heidelberg (2010)

2. Angluin, D.: Learning Regular Sets from Queries and Counterexamples. Information and Computation 75(2), 87-106 (1987)

3. Bossert, G., Hiet, G., Henin, T.: Modelling to Simulate Botnet Command and Control Protocols for the Evaluation of Network Intrusion Detection Systems. In: 2011 Conference on Network and Information Systems Security (SAR-SSI), pp. 1-8 (May 2011)

4. Cassel, S., Howar, F., Jonsson, B., Merten, M., Steffen, B.: A Succinct Canonical Register Automaton Model. In: Bultan, T., Hsiung, P.-A. (eds.) ATVA 2011. LNCS, vol. 6996, pp. 366-380. Springer, Heidelberg (2011)

5. Howar, F., Steffen, B., Cassel, S., Jonsson, B.: Inferring Canonical Register Automata. In: Kuncak, V., Rybalchenko, A. (eds.) VMCAI 2012. LNCS, vol. 7148, pp. 251-266. Springer, Heidelberg (2012)

6. Issarny, V., Steffen, B., Jonsson, B., Blair, G.S., Grace, P., Kwiatkowska, M.Z., Calinescu, R., Inverardi, P., Tivoli, M., Bertolino, A., Sabetta, A.: CONNECT Challenges: Towards Emergent Connectors for Eternal Networked Systems. In: ICECCS, pp. 154-161 (2009)

7. Lazić, R., Nowak, D.: A Unifying Approach to Data-Independence. In: Palamidessi, C. (ed.) CONCUR 2000. LNCS, vol. 1877, pp. 581-595. Springer, Heidelberg (2000)

8. Merten, M., Steffen, B., Howar, F., Margaria, T.: Next Generation LearnLib. In: Abdulla, P.A., Leino, K.R.M. (eds.) TACAS 2011. LNCS, vol. 6605, pp. 220-223. Springer, Heidelberg (2011) 